\title{
Physical and Mathematical Modelling of an Extended Nozzle for Metal Delivery to High Speed, Thin Strip Casting Machines
}

\author{
Carol JEFFERIES, Mainul HASAN and Roderick I. L. GUTHRIE
}

McGill Metals Processing Centre, Mining \& Metallurgical Engineering Department, McGill University, 3450 University St, Montreal, Quebec, Canada, H3A 2 A7.

(Received on June 5, 1995; accepted in final form on Julv 24, 1995)

\begin{abstract}
Numerical fluid flow predictions were compared with experimental flow measurements and visualization for a water model of a novel thin-strip planar-flow delivery system. The proposed slot-type planar-flow delivery system could contain a porous flow modifier and features an extended-reservoir delivery to the substrate. The experiments were carried out with water in a plexiglass model with and without the presence of ceramic foam filters. Flow visualization and velocity measurements downstream from the filter zone showed that the latter had beneficial effects in suppressing the recirculatory flow and supplying fluid of uniform velocity to the substrate. A steady, two-dimensional fluid dynamics model was developed to predict and supplement the experimental studies. This numerical model is based on the use of the Brinkman-Forchheimer extended-Darcy model in the porous filter medium and the Navier-Stokes equation in the fluid region. These two flows are coupled through the interface boundary conditions at the porous-media-fluid interface. Solutions of the coupled governing equations for the complex media and fluid regions were performed using a control-volume, finite-difference technique. The results were grid independent and converged within 2000 iterations and $30 \mathrm{~min}$ on an IBM 486. Comparison of measured fluid velocities at representative locations downstream of the filter zone showed good agreement with predicted velocities.
\end{abstract}

KEY WORDS: strip casting; delivery nozzle; flow modifier; computational fluid dynamics; water model; velocity measurements.

\section{Introduction}

Today's steel producers are currently involved in projects to reduce both operating and capital costs significantly by developing and implementing near-netshape casting processes. One of the configurations of potential interest is a novel delivery system, fitted with a porous flow modifier, for a high productivity, $13-56 \mathrm{~kg} / \mathrm{s} / \mathrm{m}$ width $(50-200 \mathrm{Tph} / \mathrm{m}$ width), horizontalbelt caster (see Fig. 1). In this planar-flow casting process, the melt is cast directly into strip by delivering the melt through a nozzle of controlled dimensions on the moving substrate which is kept chilled to a temperature below the solidus of the melt. The delivery of the melt onto the moving substrate has been identified as one of the key factors for the industrial success of a thin-strip casting process. An optimum nozzle-delivery system should supply melt to the chilled substrate with a fairly uniform velocity and without excessive mixing and turbulence generation.

In a previous paper, one of the authors has advocated the use of an extended-nozzle configuration for high speed, thin-strip, casters, for the uniform supply of low velocity melt perpendicular to a freezing substrate. ${ }^{1)}$ In one version of such a delivery system, the nozzle was fitted with an extended, reticulated, ceramic filter (or flow modifier) at its lower end. It was anticipated that such a flow modifier would dampen the high momentum of a pressure driven or gravity driven, lateral flow of metal and, by suppressing turbulence, provide a uniform, extended, pressurized, supply of low velocity liquid metal towards a horizontal substrate moving at high speed $(1-5 \mathrm{~m} / \mathrm{s})$. This represents an alternative approach to the conventional in-line, nozzle-freeding system, for which narrow gap widths and high velocities of liquid metal, require slender ceramic nozzles that have proved impractical for high-speed, thin-strip, twin-belt systems. ${ }^{2)}$

In the present work, a co-ordinated approach using numerical and physical (water) models was undertaken in order to gauge the potential value of such a system. For experimental convenience, experimental and numerical results presented here relate to water flows from an

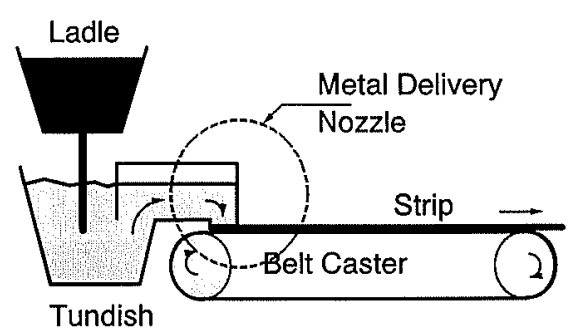

Fig. 1. Horizontal caster and delivery system. 
extended delivery nozzle towards a stationary substrate. Since the kinematic viscosities of water and liquid steel are essentially the same, the present findings on flow fields will be relevant to flows of steel for a similar configuration and flowrate, provided the effects of solidification on flows are minimal. This paper presents a comparison of fluid-flow predictions versus results from a water model of the proposed delivery system.

\section{Mathematical Model}

To describe (two-dimensional) turbulent flow into an extended metal delivery system for thin-strip casting, a mathematical model based on time-averaged equations for conservation of mass and momentum, was adopted. Associated turbulence was simplified by using an artificially enhanced viscosity.

$$
\mu_{\text {eff }}=100 \cdot \mu
$$

This ad hoc boosting of the molecular viscosity to model turbulent flow has been used by many researchers in the materials processing field, e.g. in the modelling of turbulent fluid flow and heat transfer in mould filling, ${ }^{3,4}$ ) in tundishes, and for predicting the mixing behaviour of argon stirred ladles. ${ }^{5}$ It was adopted in this model to help obviate the difficulty of modelling a region of complex media behaving as a porous medium, wherein flow would not be turbulent.

\subsection{Assumptions}

The flow was assumed to be steady, incompressible, and two dimensional. In addition, the thermophysical properties of the fluid and the porous matrix were assumed to be constant, and the porous medium was considered to be homogeneous, isotropic, and in local thermodynamic equilibrium with the fluid.

\subsection{Dimensionless Differential Equations}

In the fluid region, the momentum transport equation governing macroscopic flow was the customary NavierStokes equation. The resolved forms of this vector equation, were suitably non-dimensionalized using a two-dimensional, Cartesian system. All physical dimensions of the metal delivery system were expressed as ratios of the nozzle exit slot, $d$. Thus the following dimensionless variables were used:

$$
\begin{aligned}
& X=\frac{x}{d}, \quad Y=\frac{y}{d}, \\
& U=\frac{u \rho d}{\mu_{\mathrm{eff}}}, \quad V=\frac{v \rho d}{\mu_{\mathrm{eff}}}, \\
& P^{*}=\frac{P}{\rho}\left(\frac{d \rho}{\mu_{\mathrm{eff}}}\right)^{2}
\end{aligned}
$$

The origins of the horizontal $X$ and vertical $Y$ axes origins were located at the 'meniscus' shown on Fig. 2, at the base of the back wall. The $U$-velocities act in the $X$-direction, the $V$-velocities in the $Y$-direction. For the isothermal water model system investigated, buoyancy effects on the $V$-momentum term did not need to be considered.
The dimensionless differential equations governing the conservation of mass and momentum in the fluid region then become:

$$
\begin{array}{ll}
\text { Continuity: } & \frac{\partial U}{\partial X}+\frac{\partial V}{\partial Y}=0 \\
\text { Momentum: } & \frac{\partial U U}{\partial X}+\frac{\partial U V}{\partial Y}=\frac{\partial^{2} U}{\partial X^{2}}+\frac{\partial^{2} U}{\partial Y^{2}}-\frac{\partial P^{*}}{\partial X} \\
& \frac{\partial U V}{\partial X}+\frac{\partial V V}{\partial Y}=\frac{\partial^{2} V}{\partial X^{2}}+\frac{\partial^{2} V}{\partial Y^{2}}-\frac{\partial P^{*}}{\partial Y}
\end{array}
$$

Fluid-flow boundary conditions are summarized in Table 1. At solid-liquid boundaries there is no-slip. At the free-surfaces, the normal velocity, tangential shear stress and surface tension forces were assumed negligible. The outlet region is governed by 'free-flow' and is related to the upstream depth and nozzle exit gap and is considered to be fully-developed at the vertical transverse exit plane of the computational domain.

\subsection{Complex Media}

The extended-metal-delivery region of the proposed caster nozzle was designed to contain a reticulated ceramic filter, ${ }^{1)}$ see Fig. 2. This flow modifying region was assumed to act in a macroscopic sense, equivalent to a porous medium. The flow within the $100 \%$ fluid

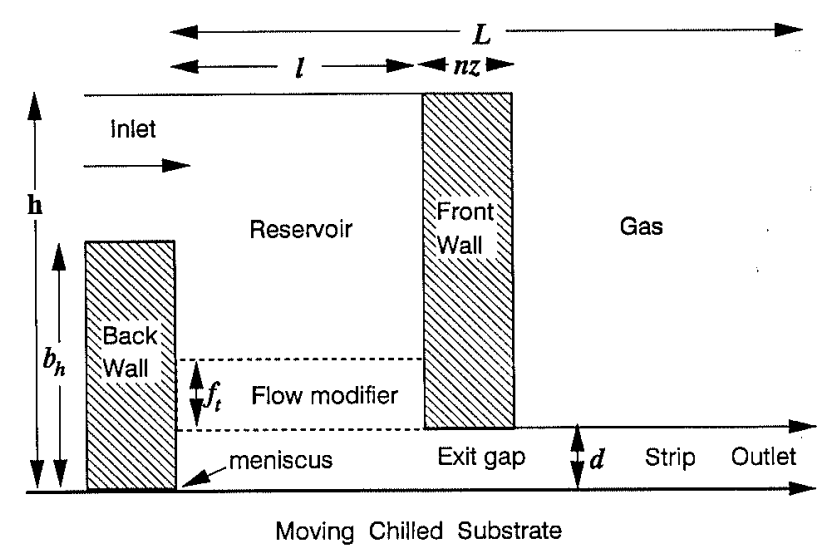

Fig. 2. Delivery nozzle schematic drawing.

Table 1. Summary of boundary conditions.

\begin{tabular}{c|cc}
\hline & $U$ momentum & $V$ momentum \\
\hline \multirow{2}{*}{ Back wall } & No slip & No slip \\
Inlet & $U=0$ & $V=0$ \\
Substrate & Uniform & $V=0$ \\
& No slip & No slip \\
Top surfaces & $U=U_{\text {sub }}=0$ & $V=0$ \\
& Stress free & No normal flow \\
Front wall & $\partial U / \partial Y=0$ & components \\
& No slip & $V=0$ \\
Outlet & $U=0$ & No slip \\
& Fully developed & $V=0$ \\
& flow & Fully developed \\
& $\partial U / \partial X=0$ & flow \\
& & $V=0$ \\
\hline
\end{tabular}


region is governed by the Navier-Stokes Eqs. (4) and (5), while flow throughout the fluid-saturated porous medium was assumed to be non-Darcian. The transport equations for the porous layer therefore incorporated the Brinkman and Forchheimer extensions to account for viscous and inertial effects, respectively. ${ }^{6-9)}$ The dimensionless governing equations for the porous layer, in terms of the superficial (Darcian) velocity are ${ }^{10,11)}$ :

$$
\begin{aligned}
& \text { Continuity: } \quad \frac{\partial U_{\mathrm{p}}}{\partial X}+\frac{\partial V_{\mathrm{p}}}{\partial Y}=0 \\
& \text { Momentum: } \quad 0=-\frac{\partial P_{\mathrm{p}}^{*}}{\partial X}+\left(\frac{\partial^{2} U_{\mathrm{p}}}{\partial X^{2}}+\frac{\partial^{2} U_{\mathrm{p}}}{\partial Y^{2}}\right) \\
& -\left(\frac{1}{D a}+\frac{\gamma\left|\overline{V E L_{\mathrm{p}}}\right|}{\sqrt{D a}}\right) U_{\mathrm{p}} \\
& 0=-\frac{\partial P_{\mathrm{p}}^{*}}{\partial Y}+\left(\frac{\partial^{2} V_{\mathrm{p}}}{\partial X^{2}}+\frac{\partial^{2} V_{\mathrm{p}}}{\partial Y^{2}}\right) \\
& -\left(\frac{1}{D a}+\frac{\gamma\left|\overline{V E L_{\mathrm{p}}}\right|}{\sqrt{D a}}\right) V_{\mathrm{p}}
\end{aligned}
$$

where, $\left|\overline{V E L_{\mathrm{p}}}\right|=\sqrt{U_{\mathrm{p}}^{2}+V_{\mathrm{p}}^{2}}$

$r=$ inertial coefficient in Forchheimer's extension $=0.55$

The Darcy number: $\quad D a=\kappa / d^{2}$

$\kappa=$ permeability of the porous medium.

In the present analysis, it was assumed that ${ }^{8)}$ :

$$
\mu_{\text {eff }, \mathrm{p}}=\mu_{\text {eff }}
$$

These two flows (fluid and complex media) are coupled through the interface boundary conditions at the complex-media-fluid interface. ${ }^{6}$ ) The governing equations for the two regions were combined into one set of conservative equations such that the fluid and the complex media were treated as a single medium with properties depending upon the location within the reservior.

The governing Eqs. (3)-(8), along with the associated boundary conditions (Table 1), were solved in the primitive variables using an implicit, control-volume, finite-difference method ${ }^{12)}$ using the SIMPLER velocitypressure correction algorithm in conjunction with the block-correction technique to speed convergence. A scalar grid system was used so that the scalar property $P$ is stored midway between the $U$ and $V$ velocity nodes. A non-uniform grid was employed, with a greater density of nodes adjacent to the solid interfaces. This grid deployment involved manual placement of the individual points.

\subsection{Grid Independence}

Grid dependency of the numerical solution was investigated. The grid density chosen was about $70 \times 50$, since this number of grid points led to momentum results that were grid independent. A non-uniform grid, as mentioned earlier, with denser packing of the points adjacent to the solid surfaces and in the exit gap, was used. A study of under-relaxation factors showed that for these highly non-linear relations, numerical convergence towards a solution was best achieved with under-relaxation factors of 0.2 for both the $U$ and $V$ velocities.

The physical properties used in the mathematical model are summarized in Table 2.

\section{Experimental Work}

A plexiglass water model with a stationary substrate was used to simulate flow in the delivery system advocated for high productivity, thin-strip casters. In designing the water modelling caster, the following factors were considered: The natural spreading thickness (i.e. height) of steel strip on a substrate is in the order of $6 \mathrm{~mm}^{13,14)}$ This height falls within the range of thickness of interest for thin strip casting operations (gauges between $3-20 \mathrm{~mm}$ thickness). The pitot tube probe had a diameter of $3 \mathrm{~mm}$, which limited the smallest gap that could be studied experimentally. The volumetric flow rates investigated are within the range corresponding to $13-56 \mathrm{~kg} / \mathrm{s} / \mathrm{m}$ width of steel $(50-200 \mathrm{Tph} / \mathrm{m})$ that are of current commercial interest for strip casters. ${ }^{15)}$

The nozzle delivery system in the water model employed a filter fitted flush to the sidewalls and exit slot. The thickness of the filter was $30 \mathrm{~mm}$, and the height of the backwall above the substrate $40 \mathrm{~mm}$. The exit slot width was $70 \mathrm{~mm}$. (See Fig. 3 for the schematic drawing of the water model.)

The water source was provided by a high pressure water line. The inlet water temperature varied between 2 and $9^{\circ} \mathrm{C}$. Microsized bubbles nucleated in the flow system owing to the lower solubility of oxygen in water at the higher laboratory temperatures. Being effectively

Table 2. Physical properties.

\begin{tabular}{c|c}
\hline Water & $\begin{array}{c}\text { Porous media } \\
\mathrm{Hi} \text { Tech ceramic, } \\
92 \% \mathrm{Al}_{2} \mathrm{O}_{3} \text { foam filters }\end{array}$ \\
\hline Density, $\rho=1000 \mathrm{~kg}^{3} \mathrm{~m}^{3}$ & $\begin{array}{c}\text { Pore size }=420 \mu \mathrm{m} \\
\text { Porosity, } \varepsilon=0.84\end{array}$ \\
Viscosity, $\mu=0.001 \mathrm{~kg} \mathrm{~m}^{-1} \mathrm{~s}^{-1}$ & $\begin{array}{c}\text { Regular density ceramic, } \\
30 \text { ppi pore density }\end{array}$ \\
$\mu_{\mathrm{ctf}}=\mu \cdot 100=0.1 \mathrm{~kg} \mathrm{~m}^{-1} \mathrm{~s}^{-1}$ & \\
$\mathrm{Kinematic}$ viscosity, & \\
$v=\mu / \rho, 10^{-6} \mathrm{~m}^{2} / \mathrm{s}$ & \\
\hline
\end{tabular}

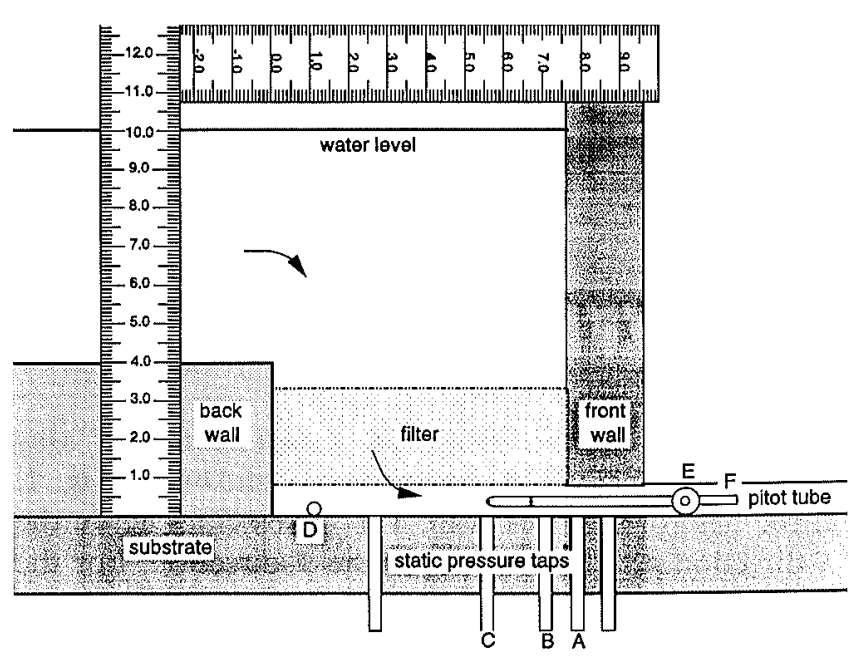

Fig. 3. Details of the reservoir zone and exit gap. 
of neutral buoyancy owing to their size, they proved to be an effective means for flow visualization. The water entered the model box via a submerged entry nozzle and then flowed through a filter followed by a honeycomb of narrow pipes so as to damp the turbulence emanating from the entry region. The narrow pipes were $76 \mathrm{~mm}$ in length and $10 \mathrm{~mm}$ in diameter. A $500 \mathrm{~mm}$ long open channel then followed. The flow into the delivery system was therefore fully developed and observed to be uniform.

Static pressures were measured at various points using inclined manometers (see the scale drawing of the delivery box; Fig. 3). Dynamic pressures were measured with the pitot tube above points A, B, and C. As noted, experiments were carried out with a stationary substrate, measurements being made some 2 to $3 \mathrm{~mm}$ above the substrate.

\subsection{Water Model Experiments}

Three exit gaps were used; 4,6 , and $8 \mathrm{~mm}$. Two entry flow conditions were considered; one with a filter (flow-modifying region), the other, without. The water level in the vessel was varied, and measurements made for heights of $80,100,120$, and $140 \mathrm{~mm}$ levels respectively. The extended nozzle measured was $76 \mathrm{~mm} \times 70 \mathrm{~mm} \mathrm{(3}$ inches by 2.75 inches). Six experimental runs were carried out as summarized in Table 3.

\subsection{Velocity and Flow Rate Measurements}

Velocity measurements were made using a stainless steel L-shaped pitot tube (United Sensor, USC-A-291-2). The diameter of the probe's nose was $3 \mathrm{~mm}$. In order to make accurate measurements in the water, it was necessary that the entire pitot tube (both compartments) be entirely filled with water. Also, all leads (tubing leading to the manometer) were completely filled with water. To accomplish this, the U-tube manometer was fitted with T's, so as to allow the bleeding of the gas from the line.

It was important to keep the pitot tube probe horizontal so that the hydrostatic effects of the filled tube would be minimized. The measuring fluid used was Meriam Blue, Specific Gravity 1.75. The height difference between the two legs of the manometer represented the dynamic pressure. As the density of water with respect to the measuring fluid was not negligible, the velocity was calculated as follows:

$$
v=\sqrt{2 \cdot g \cdot \Delta h\left(S . G \cdot{ }_{\mathrm{mf}}-S . G_{\mathbf{H}_{2} \mathrm{O}}\right.}
$$

where, $v$ : local velocity

$g$ : gravitational constant

$\Delta h$ : height difference in the manometer

Table 3. Experimental factors and levels.

\begin{tabular}{c|ccc}
\hline Exp \# & $\begin{array}{c}\text { Water levels } \\
(\mathrm{cm})\end{array}$ & $\begin{array}{c}\text { Filter } \\
(\text { Yes/No) }\end{array}$ & $\begin{array}{c}\text { Slot gap } \\
(\mathrm{mm})\end{array}$ \\
\hline 1 & $8,10,12,14$ & Yes & 4 \\
2 & $8,10,12,14$ & No & 4 \\
3 & $8,10,12,14$ & Yes & 6 \\
4 & $8,10,12,14$ & No & 6 \\
5 & $8,10,12,14$ & Yes & 8 \\
6 & $8,10,12,14$ & No & 8 \\
\hline
\end{tabular}

\section{S.G.: specific gravity}

$\mathrm{mf}$ : measuring fluid.

\subsection{Precision and Accuracy of Velocity Measurements}

For each water level in each experiment, the local velocity at three different points in the delivery system was measured. The local velocity at each of these points was repeated four times. The precision for the pitot tube measurements was estimated to be between $\pm 6-20 \%$. As well, the average flow rate, and therefore the average exit velocity, was measured using the "bucket and stopwatch" approach. The precision for an average velocity in the exit gap using this method ranged between $\pm 8-12 \%$.

A summary of observed variabilities in the various measurements is given in Figs. $\mathbf{4}$ and $\mathbf{5}$. In both figures, the standard deviation of the velocity measurements (used to calculate the sample mean velocity) is plotted against the sample mean velocity. As well as plotting the standard deviations of the measurements, the values divided by the velocity measurements (a type of non-dimensionalizing) are also plotted. It may be seen from Fig. 5 that the $\%$ variability varies from $8 \%$ for the pitot tube velocities below $1 \mathrm{~m} / \mathrm{s}$ to about $2 \%$ for those above $1 \mathrm{~m} / \mathrm{s}$. The variability in mean slot exit velocity (Fig. 4 ) is about $2 \%$ over the range of velocities studied.

To evaluate the accuracy of the measurements, the

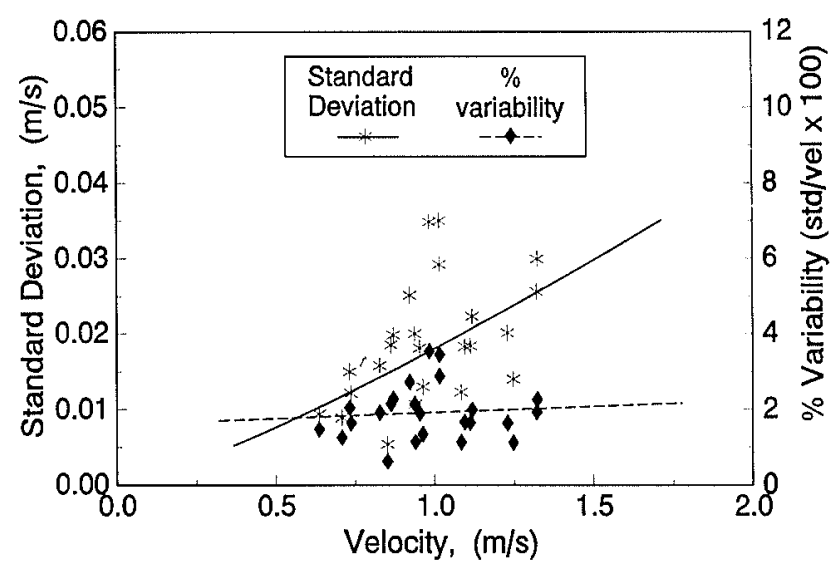

Fig. 4. Variation of the mean exit-slot velocity (bucket \& stopwatch).

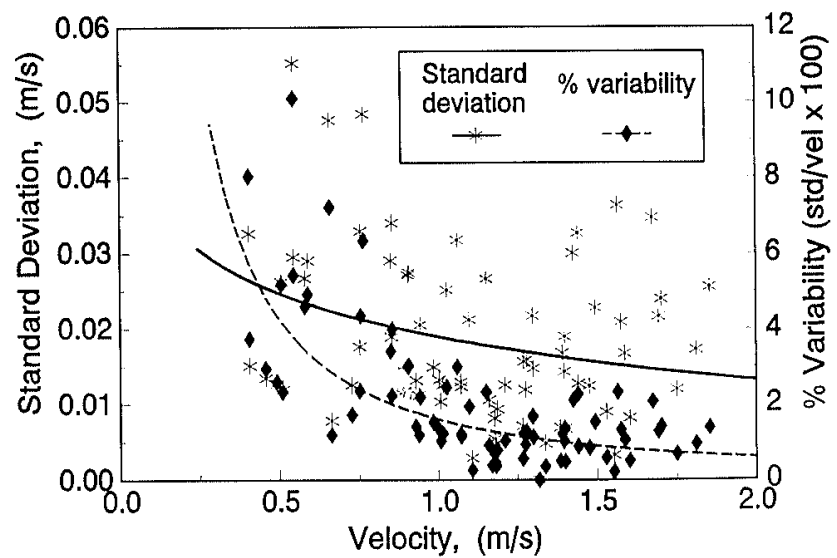

Fig. 5. Variation of local mean velocity (pitot tube measurements). 
centreline velocities measured at point $A$ (within the exit gap) were compared with measured mean velocities in the exit gap. It is well known that the ratio between average velocity and centerline velocity for laminar flow between two flat plates, is about equal to 0.5 , while for turbulent flow this ratio ranges between 0.8 and 0.9 . In the latter case, the ratio is a function of Reynolds number. The ratio's value in the range $10^{3}-10^{4}$ (exit gap Reynolds number range for these experiments) should equal $0.8 .^{16}$ )

In Fig. 6, the mean exit gap velocity $(\mathrm{m} / \mathrm{s})$, calculated from the measured volumetric flow rate, is plotted versus the centreline velocity $(\mathrm{m} / \mathrm{s})$, measured with the pitot tube in the exit gap at point $A$ and about $3 \mathrm{~mm}$ above the substrate. (The distance above the substrate for $d=$ $4 \mathrm{~mm}$ was $2 \mathrm{~mm}$, for $d=6 \mathrm{~mm}$ was $3 \mathrm{~mm}$, for $d=8 \mathrm{~mm}$ was $3.5 \mathrm{~mm}$.) These distances above the substrate were approximately the centre of the exit gap. A linear regression of the points yields a slope of 0.79 . (The slight discrepancy could in part be due to the pitot tube not being located exactly at the centreline, as well as due to experimental error.) This is the expected result and indicates that the pitot tube velocity measurements are accurate in the exit gap.

It is expected that the velocity measurements at points $\mathrm{B}$ and $\mathrm{C}$ should also be a reasonably accurate measurement of the flow impinging directly on the orifice. However, as the pitot tube is sensitive to the fluid's angle of approach, velocity measurements became less reliable. In particular, at point $C$ when no flow modifier is present, velocity measurements were less reliable, and reduced.

\subsection{Flow Visualization and Water Model Results}

There was no hydraulic jump after the exit gap. The Froude number in the exit gap ranged from 10 to 74 . The flow regime was therefore supercritical. ${ }^{17)}$ The Reynolds numbers in the exit gap based on the pitot-tube velocity measurements and using the exit gap as the characteristic length ranged between 1600 and 12800 . A summary of these ranges for the water model operation is given in Table 4.

For the experiments in which no flow modifier was present, a recirculating whorl was established adjacent to the back wall. This may be seen with the help of the

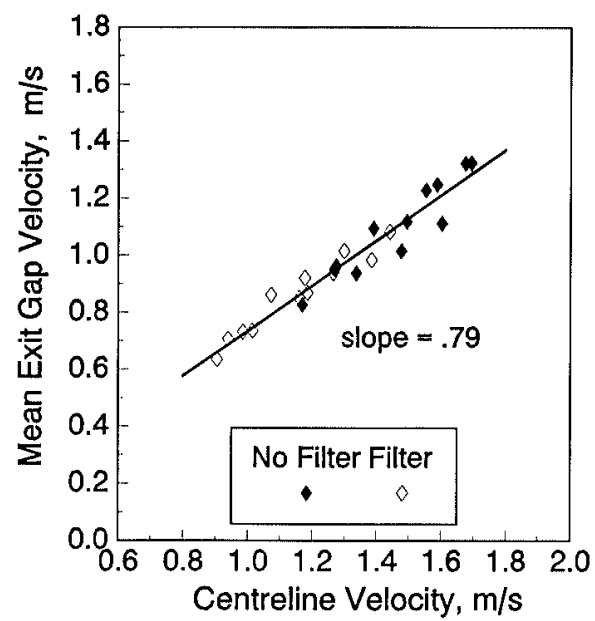

Fig. 6. The slope shows the approximate value of the ratio $V_{\text {man }} / V_{\text {max }}$. neutral buoyancy bubbles in the flow shown in Fig. 7a). Also, a dead zone is apparent close to the top front wall. For the exit gap $d=8 \mathrm{~mm}$, without the presence of a flow modifier across the reservoir, the exit gap was not completely filled with water, as for water exiting from under a sluice gate.

For experiments in which there was a flow modifier in the reservoir, the front wall dead zone was still present, but only a small recirculating eddy remained above the filter at the back wall as seen in Fig. 7b). Underneath the filter, close to the back wall the flow impinged vertically on the substrate, accelerating towards the exit gap. The porous ceramic filter had a more profound effect on flow conditions downstream from the filter, in that short circuiting of flow was virtually eliminated.

For all experiments, raising the water level in the delivery system increased the exit velocities and mass flow rate. The velocity measurements for the water model

Table 4. Ranges of Re and $F r$ numbers for points A, B, and C.

\begin{tabular}{cccc} 
Exit gap $(\mathrm{mm})$ & Filter & $\begin{array}{c}\text { Points A, B, C } \\
F r=v^{2} /(g d)\end{array}$ & $\begin{array}{c}\text { Points A, B, C } \\
\operatorname{Re}=(\rho v d) / \mu\end{array}$ \\
\hline 4 & Yes & $14-53$ & $2900-5800$ \\
4 & No & $4-84$ & $1600-7300$ \\
6 & Yes & $7-30$ & $3900-7900$ \\
6 & No & $5-58$ & $3300-11100$ \\
8 & Yes & $5-21$ & $5000-10200$ \\
8 & No & $3-33$ & $3600-12800$ \\
\hline
\end{tabular}
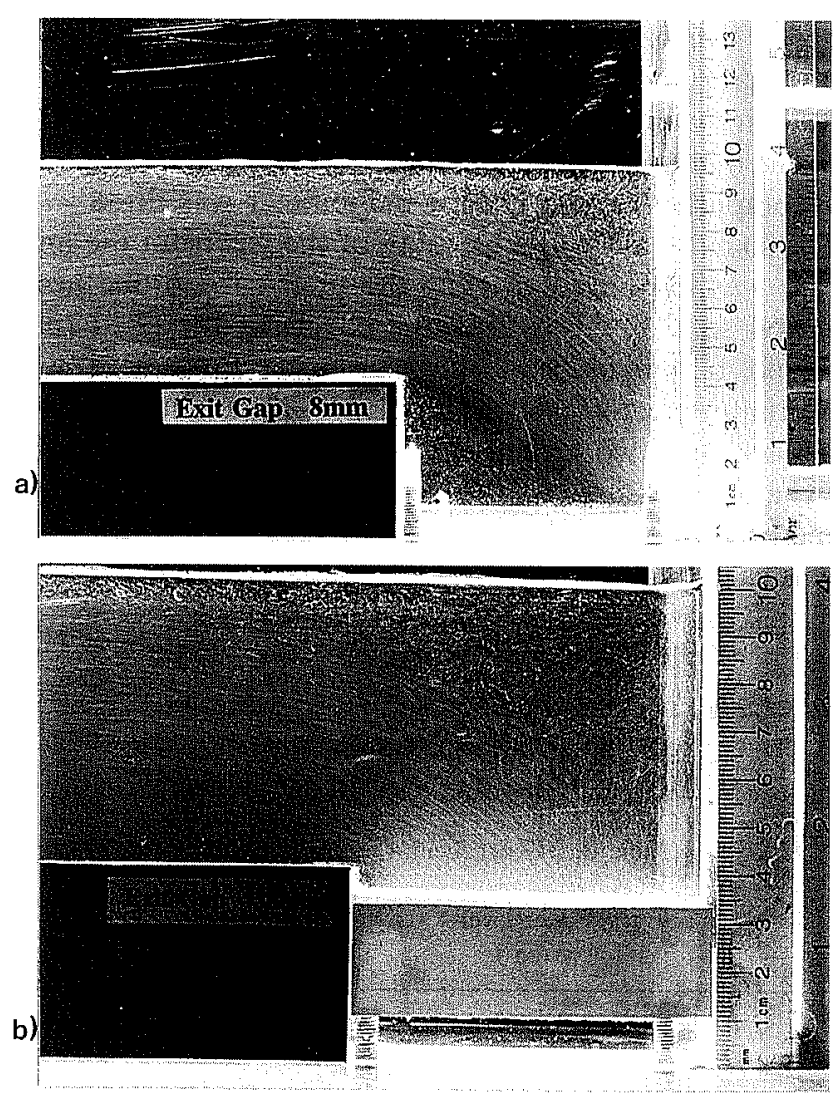

Fig. 7. a) Typical flow through the delivery zone with no filter present.

b) Typical flow through the delivery zone with the filter present. 
experiments are presented in Figs. 8 and 9 . In all cases the velocity $(\mathrm{m} / \mathrm{s})$ is plotted against the water level in millimetres. The curve fitting shows a power law relationship, but the data is in the apparently linear range and can also be fitted to a linear regression with minor differences.

In Figs. 8 and 9 the velocities for particular measurement points, A, B, and C, and the mean exit slot velocities, calculated from the volumetric flow measured by bucket and stopwatch, for all experiments, are plotted versus water level. In these figures, the asterisks represent the mean exit slot velocity measurements, the squares the measurements at position $A$, the circles the measurements at position $\mathrm{B}$ and the diamonds the measurements at position $C$. The different experiments can be distinguished by different line styles as per the legends in the upper left hand corner of each graph. Figure 8 presents the measurements made when a flow modifier was present, and Fig. 9 those made in the absence of a flow modifier. These results are compared with those generated by the mathematical model in the next section.

The presence of the flow modifier in experiments 1,3 , and 5 smooths the flow. From Figs. 8 and 9 it can be seen that the velocities measured at the different positions, $\mathrm{A}, \mathrm{B}$, and $\mathrm{C}$, when the flow modifier was present, are closer together than those velocities measured when it was absent. In particular the velocities at positions $\mathrm{A}$ and $\mathrm{B}$ (circle and squares in Fig. 8) are

\section{Flow modifier present}

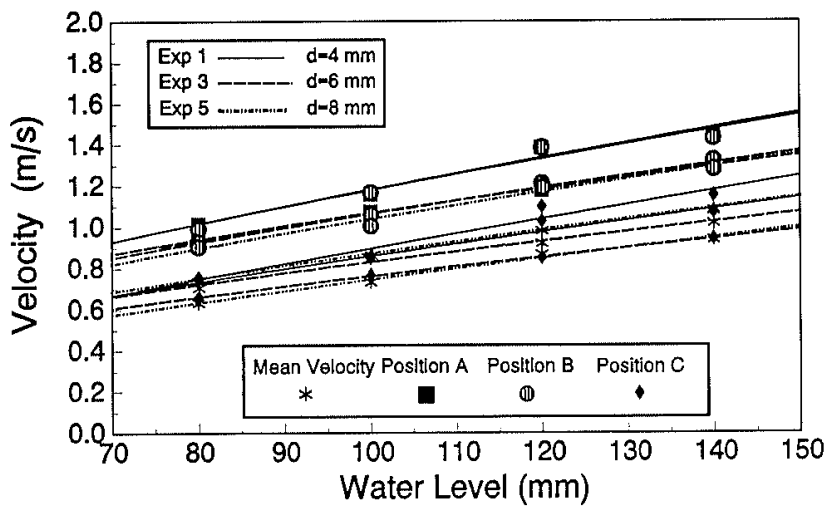

Fig. 8. Measured velocities for cases in which a flow modifier was present.

No flow modifier present.

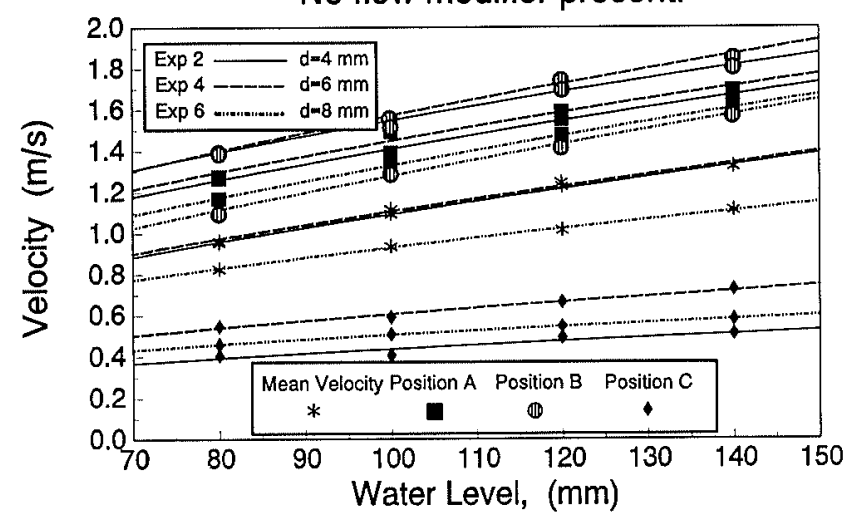

Fig. 9. Measured velocities for cases in which no flow modifier was present. practically indistinguishable for the cases for which there was flow modification. Comparing Figs. 8 and 9, it can be seen that position $\mathrm{C}$ velocities are lower for experiments 2,4 , and 6 , with no flow modification, than for experiments 1, 3, and 5 in which there are flow modifiers. This may be explained by the different flow patterns and the compression of streamlines near to the exiting gap as shown in Figs. 7a) and 7b).

The effect of the flow modifier is to raise the velocity at position $\mathrm{C}$ and lower the velocities at positions $\mathrm{A}$ and $B$ resulting in a smoother flow. As well, photographs of the "work zone" (zone of solidification) clearly show a recirculating whorl at the lower back wall in the experiments without flow modifiers (Fig. 7a)). Its presence explains the marked difference in the velocities measured at point $\mathrm{C}$ for experiments with and without flow modifiers. This recirculation and dead zone is changed when a flow modifier is present.

\section{Comparison with Numerical Model}

To test the validity of the mathematical model, computations based on the water modelling conditions were run. To do so, twenty-four simulations of the mathematical model were made, each corresponding to a different water level and experiment number. The mass flow rate corresponding to the actual water level as determined by the experiments, was used in the computer programme. In these runs, only fluid flow was considered as the water in the experiments was essentially isothermal throughout the domain. The boundary conditions are summarized in Table 1. The grid layout was such that the control volumes coincided with any solid-liquid boundaries and complex-media-liquid boundaries. The velocity of the substrate was set to zero as the physical model bottom was immobile. Figure 10 presents a summary of the average flow rates for a given water level, exit gap size and flow modification condition. Each mathematical model run corresponded to one point on Fig. 10.

\subsection{Quantitative Comparison}

The $u$-velocities predicted by the mathematical model at positions $\mathrm{A}, \mathrm{B}$, and $\mathrm{C}$ were plotted against the $u$ velocities measured in the water model at positions $A$,

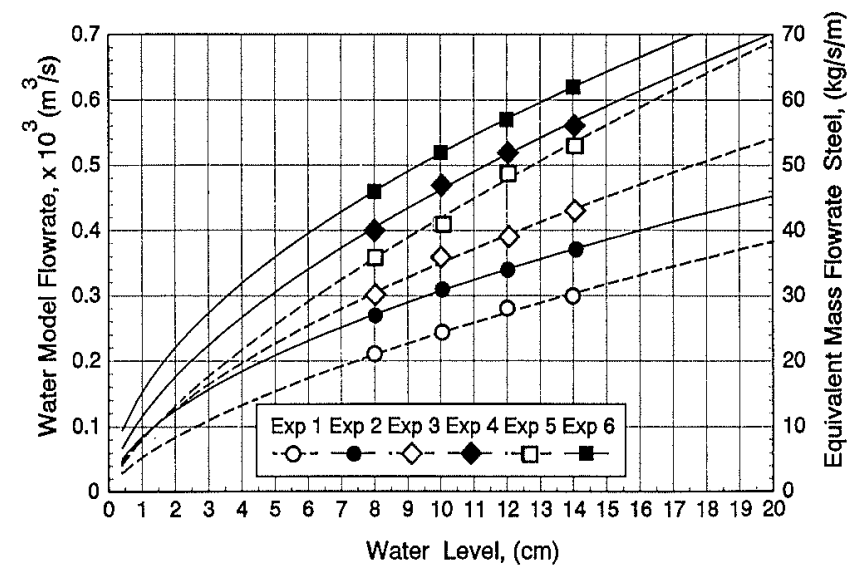

Fig. 10. Water-model flow rate versus delivery-system water level. 
B, and $C$. The results are shown in Fig. 11. The circles represent the correspondence for position $A$, the stars represent the correspondence for position $\mathrm{B}$ and the squares represent the correspondence for position $\mathrm{C}$. Different shading of the markers represents differing conditions as shown in the legend at the top left corner of the graph.

The solid line drawn on the graph represents the case of one-to-one correspondence between predicted and measured velocities. The dashed lines represent $\pm 20 \%$ correspondence, and the dotted and dashed line represent $\pm 10 \%$ correspondence. In all cases, the predictions for position $A$ fall within $\pm 10 \%$. It can be readily seen that for points $\mathrm{A}, \mathrm{B}$, and $\mathrm{C}$ in the cases in which the filter modifies the flow in the extended delivery zone, the correspondence between the two is within $\pm 20 \%$. Since the experimental error has been estimated to be between $\pm 6-20 \%$, this correspondence is good. However, for positions $\mathrm{B}$ and $\mathrm{C}$, the predictions for the cases without a flow modifier underestimate the velocities measured at these positions. In fact, the velocities predicted, for the case with no flow modification, for position B correspond within experimental error with values measured for position $\mathrm{C}$.

To help understand these comparisons, a data extraction of the computer simulations showing the region surrounding positions $\mathrm{A}, \mathrm{B}$, and $\mathrm{C}$ for the two filter conditions was made. Vector plots, of the same scale, were drawn. Figure 12 shows the velocity profiles predicted for a typical case $(d=8 \mathrm{~mm}, h=10 \mathrm{~cm})$ in which a porous flow modifying region was present, for the small region of flow near points A, B, and C. Similarly, Fig. 13 shows the profiles predicted for a typical case $(d=8 \mathrm{~mm}, h=10 \mathrm{~cm})$ in which no flow modifier was present. The main difference between these two cases was the presence or absence of the reticulate medium.

In Fig. 12, the flow can be seen to be a regular nature. The difference in the profiles for each grid point is moderate. The velocities at point B show similar values and variation with distance from the substrate to velocities at point $A$. Also, the flow is largely in the same direction. This type of flow was also more accurately measured using a pitot tube. For these cases, the correspondence between simulation values and those

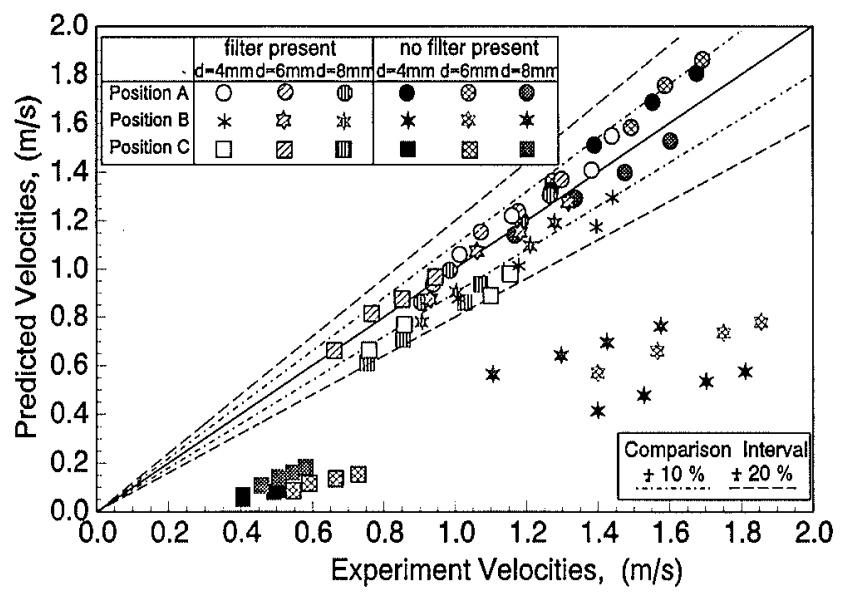

Fig. 11. Correspondence between predicted and experimental velocities. measured is, as shown on Fig. 11, good.

For Fig. 13 however, the flow is not unidirectional, and it varies quite differently in value and direction from position to position. This flow pattern corresponds qualitatively to that photographed in Fig. 7a). However, either the ad hoc viscosity treatment of turbulence by this model acts to compress the zone in which the high velocities shown may be found, and/or the pitot tube readings of velocity are less accurate owing to the oblique flows involved.

The mathematical model treatment of turbulence is a simplification and in the actual case, the effects of turbulence could act to transport the kinetic energy over a wider zone which would explain why the actual velocities at position $C$ are closer to those predicted for position $\mathrm{B}$ than those predicted for position $\mathrm{C}$. However, with the presence of a filter zone, the flow is of a more regular nature, and better corresponds to the behaviour predicted by the mathematical model (Fig. 12). It should be noted that a main objective in developing this model was the treatment of a region of porous media.

\subsection{Qualitative Comparison}

Photographs were taken of all twenty-four conditions taking advantage of the small, neutral-buoyancy bubbles to show the flow patterns. The correspondence was seen to be good. In all cases the flow lines match the predicted pattern. A few representative figures are presented here and will be discussed.

The photograph in Fig. 7b), taken for exit gap $d=8 \mathrm{~mm}$, with a filter present and water level at $10 \mathrm{~cm}$, shows a small recirculating bubble of about $10 \mathrm{~mm}$ diameter at the back wall above the filter. (Due to parallax, the exit gap appears to be $10 \mathrm{~mm}$ in the

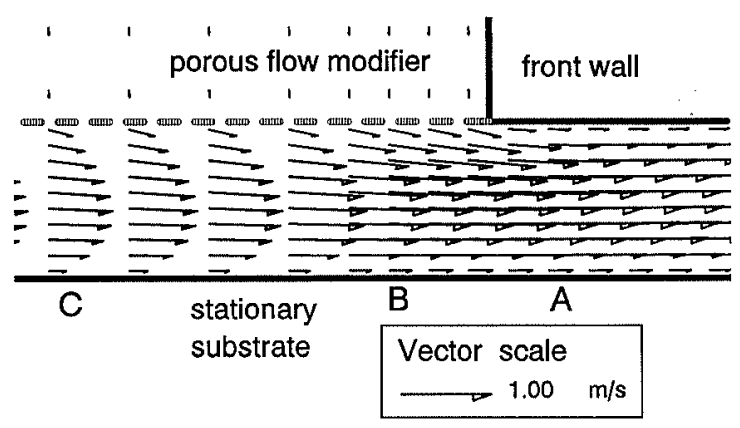

Fig. 12. Predicted velocity profiles at positions $A, B, \&$ C $(d=8 \mathrm{~mm}, h=10 \mathrm{~cm}$, with filter present $)$

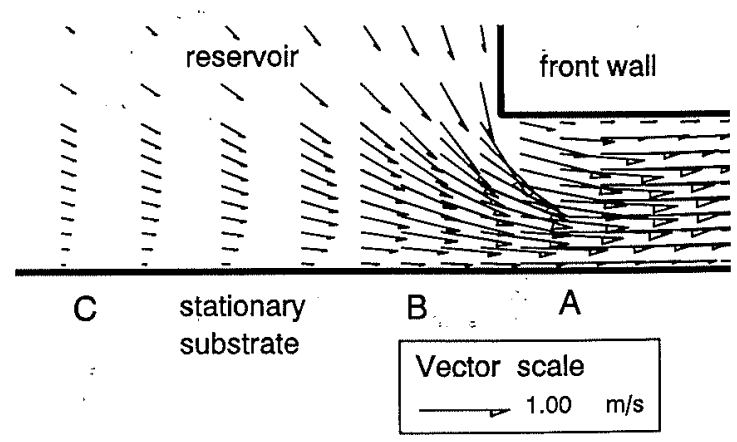

Fig. 13. Predicted velocity profiles for positions A, B, \& C ( $d=8 \mathrm{~mm}, h=10 \mathrm{~cm}$, no filter present). 
photograph.) Figure 14 showing the streamlines for the corresponding simulation shows a corresponding bending of the flow at this point. Also, the dead zone in the upper part of the reservoir close to the front wall can be clearly seen. The smooth curving of the flow lines from the side entry to their vertical flow through the filter can be clearly seen to correspond with the plotted streamlines in Fig. 14.

The photograph in Fig. 7a), taken for exit gap $d=8 \mathrm{~mm}$, with no filter present and the water level at $10 \mathrm{~cm}$, shows a slow recirculation about the height of the back wall $(\sim 4 \mathrm{~cm})$ at the back wall below the inlet. This corresponds to the streamlines predicted in this region in the corresponding simulation shown in Fig. 15. There is a dead zone in the upper part of the reservior which can be seen in both the photograph and the streamline plot. The flow lines in the photograph can be clearly seen to be diving towards, and compressing at the entrance to the exit gap as in the streamline plot of Fig. 15.

Also of interest are the velocity patterns near the domain origin. With the simulation it was possible to enlarge the vectors to see the directions of flow (Figs. 16 and 17) with, and without, flow modification. However to see the flow pattern for the case without a filter, it was necessary to enlarge the vectors by a factor of 5 times more than for the case with a filter. It can readily be concluded that, at the domain origin, in the case with no flow modifiers there is a very slow recirculation, and the feeding of fluid to that 'meniscus' is practically nonexistent.

\section{Conclusions}

The presence of the reticulate medium smooths flows across the substrate. The velocities at points $\mathrm{A}, \mathrm{B}$, and $C$ were then more uniform (that is, closer together) and there were no recirculating zones under the filter. In particular, the flow modifiers had a marked effect on the velocities at point $C$ (raising them) and they moderated the velocities at points $\mathrm{A}$ and $\mathrm{B}$ (lowering them). It was possible to obtain repeatable measurements of the local velocity with the pitot tube. The values for the centreline velocities in the exit gap corresponded to what would be expected for turbulent flow based upon the average velocities measured.

The model is capable of simulating the actual hydrodynamic behaviour of the extended-delivery zone. The quantitative results show a good correspondence for all the cases in which a flow modifier is present. In every comparison of the velocities at point $\mathrm{A}$, the correlation is good. Qualitatively the predictions for every case match the observed flow patterns. This includes those cases with no flow modifier present.

The model predicts a poor feeding of fluid to the lower back wall when there is no flow modification. For a hot metal system this dead zone would usually lead to the attachment of a skull at the meniscus. A better feeding mechanism is apparent when the flow modifier is present.

Now that this model has been validated, it can be used to simulate the effect of a rapidly moving substrate on fluid flow and will be especially useful when examining regions which may be difficult to see, or regions in which it may be difficult to make measurements. This information can be useful to predict behaviour such as the uneven

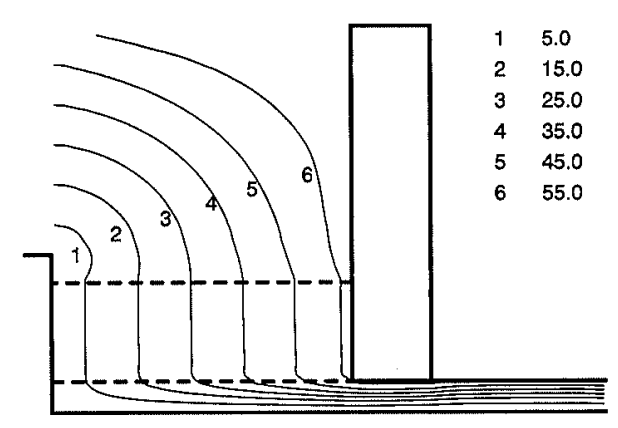

Fig. 14. Dimensionless stream-function contours predicted for flow through the delivery zone with flow modification $(d=8 \mathrm{~mm}, h=10 \mathrm{~cm})$.

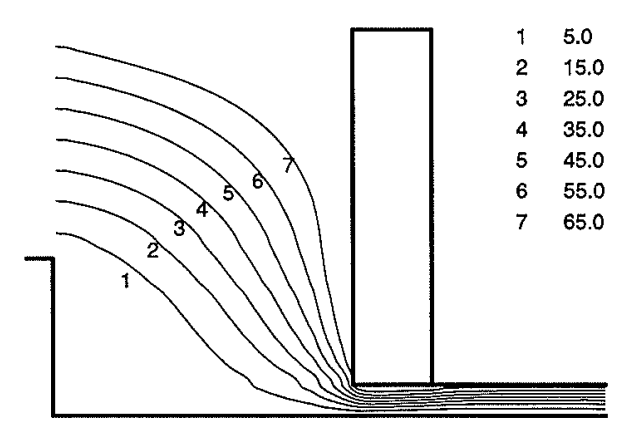

Fig. 15. Dimensionless stream-function contours predicted for flow through the delivery zone in the absence of flow modification $(d=8 \mathrm{~mm}, h=10 \mathrm{~cm})$.

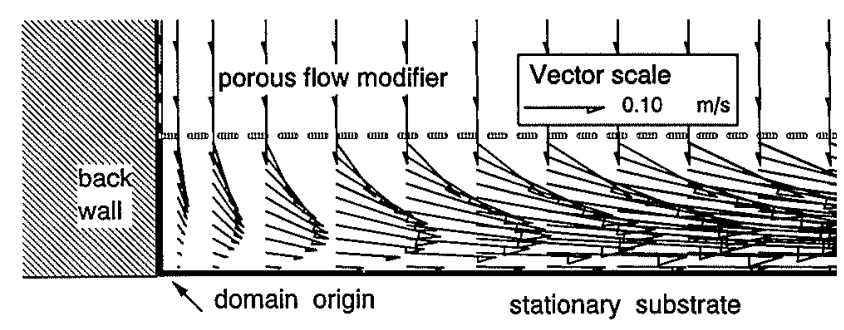

Fig. 16. Velocity vectors near the origin for exit gap $d=8 \mathrm{~mm}$, with a filter present.

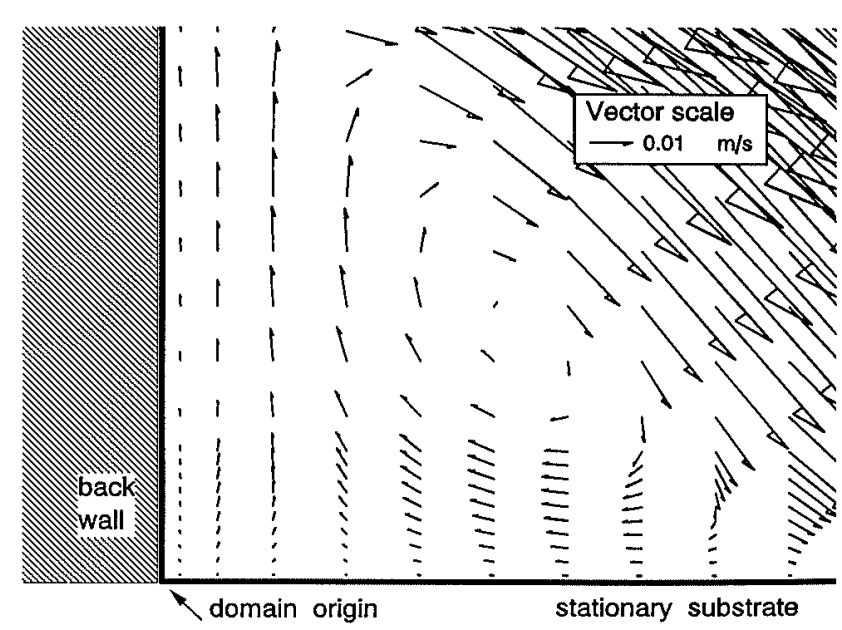

Fig. 17. Velocity vectors near the origin for exit gap $d=8 \mathrm{~mm}$, with no filter present. 
feeding of liquid metals with its concomitant problems of shell remelting and skull attachment.

\section{Acknowledgements}

The authors would like to acknowledge the NSERC operating grant system for making this work possible as well as the cooperation of BHP for their input into the formulation of the problem.

\section{REFERENCES}

1) J. Herbertson and R. I. L. Guthrie: Proc. of an Int. Symp. on Casting of Near Net Shape Products, The Metallurgical Soc., Inc., Hawaii, (1988), 335.

2) S. S. Daniel, P. E. Hamill, A. Vassilicos, J. H. Baker, T. W. Lewis and C. D. Dykes: Proc. of Int. Conf. on New Smelting Reduction and Near Net Shape Casting Technologies for Steel (SRNC-90), Pohang, Korea, (1990), 483.

3) S. Mishima and J. Szekely: ISIJ Int., 29 (1989), 324.

4) K. S. Chan, K. Pericleous and M. Cross: Appl. Math. Model., 15 (1991), 624 .

5) D. Mazumdar and R. I. L. Guthrie: Appl. Math. Model,, 10 (1986), 25.
6) C. Jefferies, M. Hasan and R. I. L. Guthrie: Proc. of 29 th C. I. M. Conference, Hamilton, (1990), 313

7) C. Beckermann, R. Viskanta and S. Ramadhyani: Numer. Heat Transfer, 10 (1986), 557.

8) C. Beckermann, R. Viskanta and S. Ramadhyani: J. Fluid Mech., 186 (1988), 275

9) R. C. Givler and S. A. Altobelli: J. Fluid Mech., 258 (1994), 355.

10) N. Kladias and V. Prasad: J. Heat Transfer, 111 (1989), 926.

1I) V. Prasad, M. Keyhani and R. Shen: J. Electron. Packaging, 112 (1990), 63

12) S. V. Patankar: Numerical Heat Transfer and Fluid Flow, Hemisphere Publishing Corporation, New York, (1980).

13) J. Herbertson, J. C. Campbell, A. G. Hunt and J. Freeman: Proc. of 5th Int. Conf. on Continuous Casting, Linz, (1990).

14) P. C. Campbell and N. Blake: BHP Central Research Laboratories, Technical Note, (1990).

15) R. Nyström, E. Burström, W. Reichelt and U. Urlau: Metec 94 , 2nd European Conf. on Continuous Casting, Düsseldorf, (1994).

16) J. W. Dally, W. F. Riley and K. G. McConnell: Instrumentation for Engineering Measurements, Wiley, New York, (1984), 473.

17) J. A. Roberson and C. T. Crowe: Engineering Fluid Mechanics, Houghton Mifflin Co., (1975). 